\title{
The safety, efficacy, and pharmacokinetics of esmolol for blood pressure control immediately after repair of coarctation of the aorta in infants and children: A multicenter, double-blind, randomized trial
}

Sarah Tabbutt, MD, PhD, ${ }^{a}$ Susan C. Nicolson, MD, ${ }^{\text {a }}$ Peter C. Adamson, MD, ${ }^{a}$ Xuemei Zhang, MS, ${ }^{a}$ Marc L. Hoffman, MD, ${ }^{9}$ Winfield Wells, MD, ${ }^{\text {b }}$ Carl L. Backer, MD, ${ }^{c}$ Francis X. McGowan, MD, ${ }^{d}$ James S. Tweddell, MD, ${ }^{e}$ Paula Bokesch, MD, ${ }^{f}$ and Mark Schreiner, MD

Earn CME credits at http:// cme.ctsnetjournals.org
From the Children's Hospital of Philadelphia, ${ }^{a}$ Philadelphia, Pa; Los Angeles Children's Hospital, ${ }^{\mathrm{b}}$ Los Angeles, Calif; Children's Memorial Hospital, ${ }^{\mathrm{c}}$ Chicago, Ill; Children's Hospital of Boston, ${ }^{\mathrm{d}}$ Boston, Mass; Wisconsin Children's Hospital, ${ }^{\mathrm{e}}$ Milwaukee, Wis; Cleveland Clinic Foundation, ${ }^{\mathrm{f}}$ Cleveland, Ohio; and Baxter Healthcare, ${ }^{\mathrm{g}}$ New Providence, NJ.

Supported by Baxter Healthcare Corporation.

Received for publication Aug 16, 2007; revisions received Sept 5, 2007.

Address for reprints: Sarah Tabbutt, MD $\mathrm{PhD}$, Cardiac Intensive Care Unit, the Children's Hospital of Philadelphia, 34th Street and Civic Center Blvd, Philadelphia, PA 19104 (E-mail: tabbutt@email.chop.edu).

J Thorac Cardiovasc Surg 2008;136:321-8 0022-5223/\$34.00

Copyright $(\odot 2008$ by The American Association for Thoracic Surgery

doi:10.1016/j.jtcvs.2007.09.086
Objectives: Blood pressure control is important after repair of coarctation of the aorta. We report the first prospective multi-institutional trial addressing the safety and efficacy of esmolol after repair of coarctation of the aorta in infants and children.

Methods: The primary objective of this phase IIIb, multicenter, double-blind, randomized, dose-ranging trial was the efficacy of esmolol to control hypertension. Candidates included subjects younger than 6 years and weighing $2.5 \mathrm{~kg}$ or more who underwent surgical intervention for coarctation of the aorta and required therapy for systemic hypertension. One hundred sixteen subjects received esmolol: 36 received a low dose $(125 \mu \mathrm{g} / \mathrm{kg}), 43$ received a medium dose $(250 \mu \mathrm{g} / \mathrm{kg})$, and 37 received a high dose $(500 \mu \mathrm{g} / \mathrm{kg})$. The primary outcomes were the change in systolic blood pressure and the need for additional antihypertensive rescue medication $5 \mathrm{~min}$ utes after the initiation of esmolol.

Results: All dose groups showed a significant decrease from baseline in systolic blood pressure $(-9.6 \pm 16.3 \mathrm{~mm} \mathrm{Hg}, P<.001)$. There were no differences in systolic blood pressure response at 5 minutes between dose groups (high, medium, or low) or age groups. The need for rescue medication at 5 minutes was not different between dose groups. All dose groups showed similar incidences of adverse events. There were no serious adverse events.

Discussion: Esmolol can be administered safely to patients younger than 6 years after repair of coarctation of the aorta. In the dose range of 125 to $500 \mu \mathrm{g} / \mathrm{kg}$, esmolol significantly decreased systolic blood pressure.

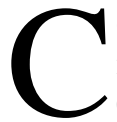

oarctation of the aorta $(\mathrm{CoA})$ comprises approximately $6 \%$ of all congenital heart disease. ${ }^{1}$ The surgical mortality of isolated coarctation is low at $0.5 \%$ to $2.5 \%{ }^{2,3}$ Hypertension occurs in more than $65 \%$ of subjects during the postoperative period after surgical CoA repair. ${ }^{4-6}$ The short-acting and selective $\beta_{1}$-antagonist esmolol hydrochloride (esmolol) has been shown to achieve early and predictable steady-state blood levels and to have a short duration of action. ${ }^{7,8}$ Esmolol has been shown to control hypertension in children after cardiac surgery ${ }^{9}$ and specifically after CoA repair (in both case reports ${ }^{10}$ and small series $\left.^{9,11}\right)$. This article is the first multicenter report of the dose response and safety of esmolol for the treatment of hypertension in infants and children after CoA repair. The primary aim was 2-fold: (1) the change in blood pressure from baseline and (2) the need for antihypertensive rescue medications. The secondary aims were (1) dose dependence, (2) age dependence, (3) pharmacokinetics, and (4) safety assessment. 


\author{
Abbreviations and Acronyms \\ $\mathrm{AE} \quad=$ adverse event \\ ANOVA $=$ analysis of variance \\ $\mathrm{CoA}=$ coarctation of the aorta \\ FDA $=$ US Food and Drug Administration \\ SBP = systolic blood pressure
}

\section{Materials and Methods}

This phase IIIb, multicenter, double-blind, randomized, active control, dose-ranging study was conducted at 20 institutions in North America; the institutional review board at each site approved the study. This study (protocol no. 20,015-004 entitled "A multi-center study to evaluate the efficacy, safety and pharmacokinetics of esmolol hydrochloride (Brevibloc) for treatment of hypertension in infants and children after surgical repair of coarctation of the aorta") was approved by the US Food and Drug Administration's (FDA) Division of Cardio-Renal Drug Products and monitored by the FDA's Bioresearch Monitoring Program. The steering committee, composed of cardiac anesthesiologists and cardiac intensivists from 3 high-volume pediatric centers (Children's Hospital of Philadelphia, Boston Children's Hospital, and Cleveland Clinic), assisted in the study design to ensure consistency with standard of care. Pediatric patients younger than 6 years and weighing $2.5 \mathrm{~kg}$ or more scheduled for surgical repair of $\mathrm{CoA}$ through a lateral thoracotomy were screened for eligibility. Participation in the study required a 2stage eligibility process. Patients were evaluated in the preoperative phase to determine whether they were eligible for enrollment. Patients meeting screening criteria and for whom informed consent was obtained were randomized before surgical intervention. The final inclusion criteria were determined based on systolic blood pressure (SBP) after aortic crossclamp release.

Preliminary exclusion criteria included any of the following: (1) an awake resting heart rate of less than 120 beats/min for a neonate ( $\leq 28$ days), less than 90 beats/min for an infant ( $>28$ days to $<1$ year), or less than 70 beats/min for a child ( $\geq 1$ year to 6 years); (2) reactive airway disease that required any form of therapy within the previous week or required hospital admission within the past year; (3) a known sensitivity to $\beta$-blockers; (4) hypoglycemic presentation at the time of screening evaluation; (5) participation in a randomized study or exposure to any experimental drug within 28 days before enrollment; (6) concomitant complex congenital heart disease requiring cardiopulmonary bypass; (7) shock, acidosis, and/or decreased left ventricular function within 48 hours of surgical intervention; (8) abnormal clinical laboratory values; (9) previous treatment in the study; or (10) on-going treatment with a $\beta$-blocker or long-acting antihypertensive agent. Final inclusion criteria were hypertension that required treatment (neonate, $\mathrm{SBP} \geq 80 \mathrm{~mm} \mathrm{Hg}$; infant, $\mathrm{SBP} \geq 85 \mathrm{~mm} \mathrm{Hg}$; child, $\mathrm{SBP} \geq 95 \mathrm{~mm} \mathrm{Hg}$ ) within 30 minutes of crossclamp release and no prior intraoperative antihypertensive treatment, with the exception of nitroprusside, before crossclamp release. Nitroprusside use was permitted during surgical intervention provided it was stopped at least 2 minutes before crossclamp release and at least 10 minutes before the time the study drug was to be administered. The subject must also have not received any inotropic agent for the treatment of hypotension after crossclamp release.

\section{Interventions}

The steering committee determined that a placebo-controlled design was not feasible because of the need to rapidly control blood pressure in this subject population, and thus a dose-ranging design was used. The dose range was determined by the steering committee to span the standard of care. Subjects were randomly assigned to receive a low dose $(125 \mu \mathrm{g} / \mathrm{kg})$, medium dose $(250 \mu \mathrm{g} / \mathrm{kg})$, or high dose $(500 \mu \mathrm{g} / \mathrm{kg})$. An initial bolus dose of either 125,200 , or $500 \mu \mathrm{g} / \mathrm{kg}$ was administered over 10 to 20 seconds into a free-running designated intravenous line, followed by continuous infusion, at the same minutely rate, maintained at a rate of $1.5 \mathrm{~mL} \cdot \mathrm{kg}^{-1} \cdot \mathrm{h}^{-1}$ for at least 15 minutes to complete the primary efficacy assessments and sampling for pharmacokinetic analysis. Rescue medication was permitted 5 minutes after the loading dose if the subject's SBP had not decreased to less than the threshold for study drug administration. The choice and dose of antihypertensive rescue medication was at the discretion of the anesthesiologist and surgeon. After the 15-minute dose-response phase was completed, blood pressure management could be accomplished by (1) increasing or decreasing the blinded esmolol administration rate, (2) adding unblinded esmolol to the blinded esmolol infusion, or (3) adding any additional antihypertensive treatments the investigator deemed appropriate. Blinded esmolol administration was permitted for up to 12 hours, after which open-label esmolol could be continued at the discretion of the investigator.

\section{Objectives/Outcomes}

The primary end points were (1) change in SBP and (2) the percentage of subjects requiring rescue medication 5 minutes after the initiation of esmolol. Efficacy parameters included the change in SBP at 5 minutes when comparing age groups and dose groups. Heart rate and blood pressure during anesthesia were measured with a right upper extremity arterial catheter. Blood pressures were measured with an arterial catheter until the catheter was removed, after which noninvasive automated blood pressures were obtained. Heart rate and blood pressure were measured immediately before administration of the study drug loading dose and at 1-minute intervals until 15 minutes after the loading dose. The secondary objective was to determine the pharmacokinetics of esmolol when administered as an infusion after a loading dose and to asses the tolerability and safety of esmolol infusion in infants and children after repair of CoA.

\section{Randomization}

A centrally administered, balanced, computerized, dynamic randomization scheme was used for the randomization of subjects to dose groups. Subjects who were randomized but who did not become hypertensive within the protocol-specified timeframe were considered screen failures. Randomized subjects who did not receive study drug or were not evaluable for efficacy were replaced through the dynamic randomization scheme. The system verified that the difference between any 2 treatment groups never varied by more than 2 subjects.

Clinicians and clinical research personnel, including medical monitors, site monitors, and statisticians, were kept blinded to the dose of study drug until the conclusion of the study.

\section{Data Quality Assurance}

Sponsor clinical research associates performed initiation and routine monitoring visits to all 20 sites. Data from case report forms were 
double entered into the clinical study database (ClinTrial; Phase Forward, Inc, Waltham, Mass). Data queries were returned by clinical research associates and resolved by individual sites.

\section{Safety Reporting and Adverse Events}

Adverse events (AEs) were collected and rated by severity and relationship to study drug by the investigators. AEs were summarized overall and by sex, race, age group, and concealed dose groups. These data were made available to the Date Safety Monitoring Board and individual institutional review boards. Two interim analyses of AE data were performed after 30 and 60 patients were enrolled. Investigational New Drug (IND) Safety Reports were sent to the FDA and to all individual institutional review boards.

\section{Statistical Methods}

Data analyses were performed by Baxter Healthcare Corporation (Deerfield, Ill) in conjunction with statisticians at the Children's Hospital of Philadelphia (XZ). SAS Version 8.1 for Windows (SAS, Inc, Cary, NC) was the primary statistical software used. All statistical tests, except the primary end points, were based on a 2-tailed test at a .05 significance level. There were 5 subsets of data analyzed for this study: (1) all patients who randomized, regardless of treatment with esmolol; (2) safety, randomized patients who received any esmolol; (3) intent-to-treat efficacy, randomized patients who received esmolol and had a blood pressure measurement at 5 minutes after loading dose or earlier if hypotensive; (4) per-protocol efficacy, patients in intent-to-treat efficacy who did not have major protocol violations; and (5) pharmacokinetics, randomized patients who received esmolol and had valid drug concentration data. The intent-to-treat and per-protocol populations were used in the efficacy analysis.

There were 2 primary efficacy outcome measures: (1) the change in SBP and (2) the need for rescue medication. For the primary efficacy outcome, the change from baseline in SBP measured before the esmolol loading dose and at 5 minutes after the loading dose and the differences in the 3 dose groups were compared by using a 1-way analysis of variance (ANOVA), with the dose groups as the factor levels. Tukey pairwise comparisons were performed when statistical significance was found to establish which dose groups had similar or different effects. The primary efficacy outcome of the percentage of patients requiring rescue medication within 5 minutes after starting the esmolol infusion was determined by using 2 methods. The first analysis was a 1-sided Cochran-Armitage linear trend test with the null hypothesis that the percentage of patients requiring rescue medication was comparable across all 3 dose groups. The second analysis hypothesized that there was an order between the percentage of patients requiring medication and the decrease in percentage of rescue medication with increased dose. This was evaluated by using an exact $\chi^{2}$ test comparing the percentages of patients requiring rescue medication.

The secondary efficacy parameter used a 2-way ANOVA analysis for change in SBP at 5 minutes, with age groups being the secondary factor to account for the different blood pressure thresholds for entry and exclusion from the study for each age group. Additional outcome measures of diastolic blood pressure, mean arterial pressure, and heart rate were investigated. One-way ANOVA for change from baseline at 5 minutes was used, with the dose groups as the factor levels.
For safety analysis, AEs were summarized as the percentage of patients with any $\mathrm{AE}$ and by each specific $\mathrm{AE}$ and system organ class overall and by dose group.

\section{Pharmacokinetics-pharmacodynamics}

Blood samples of 1 to $1.5 \mathrm{~mL}$ were taken from the arterial line before the loading dose was given and at 5, 10, and 15 minutes after the loading dose. All blood samples were processed, stored, and shipped to the reference laboratory (Division of Clinical Pharmacology and Therapeutics at The Children's Hospital of Philadelphia) and analyzed for plasma esmolol concentration by using a previously published method. ${ }^{12}$ Because a sparse sampling was used for the study, pharmacokinetic analyses were performed by using data from the population. Patients with documented problems or errors in drug administration were excluded from pharmacokinetic analyses. Clearance was calculated by dividing the infusion rate by the steady-state concentration. The half-life of accumulation was determined by dividing 0.693 by the rate constant. A regression analysis of steady-state concentration $\left(\mathrm{C}_{\mathrm{ss}}\right)$ versus infusion rate was performed to define whether the pharmacokinetics of esmolol over the patient age range were linear.

\section{Protocol Amendment}

There was one protocol amendment made during this study. The analysis plan was modified and the sample size was increased per a request from the FDA. The protocol was revised to perform the primary efficacy analyses by using the assumption that the 2 primary end points were correlated and to perform the analysis of the percentage of subjects requiring rescue medication as a 2 -tailed test. This revision resulted in an increased sample size of $20 \%$, from 90 efficacyevaluable subjects (with 120 randomized to account for dropouts).

\section{Results}

Eligible participants were recruited from August 2000 until November 2002. Subjects were screened preoperatively and participated in the study from the time of induction of anesthesia until hospital discharge. One hundred sixty-four subjects consented to participate in the study (Figure 1). Of the subjects randomized, 118 met the final inclusion criteria for study participation and received esmolol, 36 in the lowdose $(125 \mu \mathrm{g} / \mathrm{kg}), 44$ in the medium-dose $(250 \mu \mathrm{g} / \mathrm{kg})$, and 38 in the high-dose $(500 \mu \mathrm{g} / \mathrm{kg})$ groups. Of the 118 subjects treated with esmolol, 116 were included in the intent-to-treat subset as the primary end point (blood pressure at 5 minutes could not be measured for 2 subjects). One hundred ten subjects were included in the per-protocol efficacy subset because 6 subjects had protocol violations: incorrect loading dose $(n=2)$, incorrect infusion rate $(n=2)$, delay in continuous infusion $(\mathrm{n}=1)$, and nipride administered before 5 minutes after blinded study drug administration $(\mathrm{n}=1)$. All other deviations did not adversely affect the ability to interpret the study efficacy or safety data and were considered minor. Blood samples for pharmacokinetic analysis were available for 117 subjects; 107 were interpretable. The safety subset was used for safety analysis and included all 118 subjects who were treated with any esmolol. 


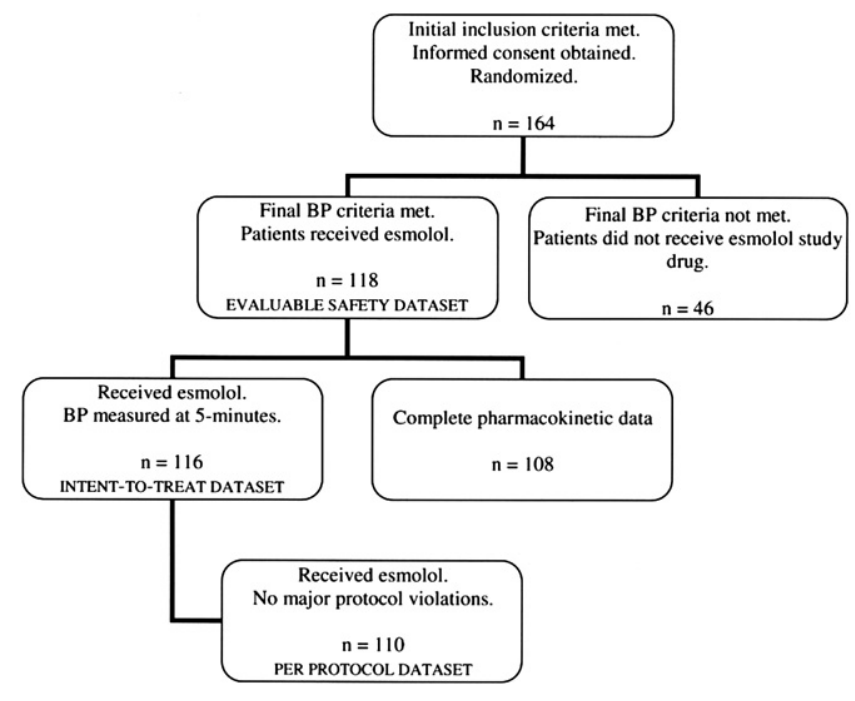

Figure 1. Flow diagram of patients in whom informed consent was obtained and randomization occurred. BP, Blood pressure.

There were no significant differences between the dose groups with respect to their medication history, prestudy physical examination findings, or medical history abnormalities (Table 1).

\section{Primary Outcomes}

There was a significant decrease in SBP at 5 minutes in all dose groups compared with baseline values (Table 2). No statistical difference was found between dose groups. There was no difference between dose groups in the change ( $P=$ .24) from baseline in SBP. In the intent-to-treat subset, 63
(54\%) of the subjects met the criteria for rescue medication after 5 minutes of blinded esmolol. Two analyses were performed on these data: an exact $\chi^{2}$ test to verify that the proportions of patients who met the criteria for and who were given rescue medications were the same and a Cochran-Armitage linear trend test to determine whether a trend existed across the dose groups. There were no statistical differences found (Table 2). Thirty-nine (62\%) of the patients who met the criteria actually received rescue medication at 5 minutes after the start of esmolol. The types of rescue medications included increased isoflurane, nitroprusside, fentanyl, and propofol. Some patients received more than 1 agent.

\section{Secondary Outcomes}

There was no statistical difference in SBP response between age groups (neonate, infant, and child; $P=.32$ ). There were no statistical differences between dose groups for change from baseline in diastolic blood pressure or mean arterial pressure at 5 minutes after esmolol administration. There was a statistical difference seen in change from baseline in mean heart rate 5 minutes after esmolol administration for the per-protocol efficacy analyses and a trend toward significance in the intent-to-treat analyses (Table 3). There was no relationship between percentage decrease in SBP at 5 minutes and the simultaneous arterial plasma esmolol concentration. Subjects' heart rate decreased at 5 minutes with increasing arterial plasma esmolol concentration $(r=$ 0.41).

Esmolol was discontinued when it was no longer believed to be needed because of hemodynamic stability $(\mathrm{n}=75$ [68\%]), inadequate blood pressure control $(n=6[6 \%])$, AEs $(n=7[6 \%])$, or “other" $(n=22[20 \%])$.

TABLE 1. Demographics*

\begin{tabular}{|c|c|c|c|c|}
\hline & Low dose & Medium dose & High dose & Overall \\
\hline $\mathrm{N}$ & 36 & 44 & 38 & 118 \\
\hline \multicolumn{5}{|l|}{ Age $(\mathrm{mo})$} \\
\hline Mean (SD) & $18.7 \pm 23.0$ & $19.4 \pm 24.5$ & $14.6 \pm 19.4$ & $17.6 \pm 22.4$ \\
\hline \multicolumn{5}{|l|}{ Sex $(n)$} \\
\hline Male/female & $21 / 15$ & $32 / 12$ & $26 / 12$ & $79 / 39$ \\
\hline \multicolumn{5}{|l|}{ Race (n [\%]) } \\
\hline White & $26(72 \%)$ & $33(75 \%)$ & $25(66 \%)$ & $84(71 \%)$ \\
\hline Hispanic & $5(14 \%)$ & $9(20 \%)$ & $11(29 \%)$ & $25(21 \%)$ \\
\hline Black & $3(8 \%)$ & $2(5 \%)$ & $2(5 \%)$ & $7(6 \%)$ \\
\hline Other/unknown & $2(6 \%)$ & $0(0 \%)$ & $0(0 \%)$ & $2(2 \%)$ \\
\hline \multicolumn{5}{|l|}{ Weight (kg) } \\
\hline Mean (SD) & $9.3 \pm 6.5$ & $9.5 \pm 6.2$ & $8.4 \pm 5.5$ & $9.1 \pm 6.0$ \\
\hline \multicolumn{5}{|l|}{ Age group (n [\%]) } \\
\hline$\leq 28$ days & $9(25 \%)$ & $12(27 \%)$ & $5(13 \%)$ & $26(22 \%)$ \\
\hline$>28 \mathrm{~d}$ to $<1 \mathrm{y}$ & $14(39 \%)$ & $13(30 \%)$ & $18(47 \%)$ & $45(38 \%)$ \\
\hline$\geq 1 \mathrm{y}$ to $6 \mathrm{y}$ & $13(36 \%)$ & $19(43 \%)$ & $15(39 \%)$ & $47(40 \%)$ \\
\hline
\end{tabular}

$S D$, Standard deviation. *Safety dataset: all patients receiving study esmolol. 
TABLE 2. Primary end point evaluation (intent-to-treat subset)

\begin{tabular}{|c|c|c|c|c|c|c|}
\hline Parameter & Statistic/category & Low dose & Medium dose & High dose & Overall & $P$ value \\
\hline \multicolumn{7}{|c|}{ Change from baseline in systolic blood pressure within 5 min after esmolol start } \\
\hline $\mathrm{N}$ & & 36 & 43 & 37 & 116 & \\
\hline Change from baseline $(\mathrm{mm} \mathrm{Hg})$ & Mean (SD) & $-6.0 \pm 12.6$ & $-12.2 \pm 16.8$ & $-10.2 \pm 18.7$ & $-9.6 \pm 16.3$ & .24 \\
\hline Tests for change from baseline equal to 0 & $\mathrm{~T}(P$ value $)$ & $-2.9(<.01)$ & $-4.8(<.001)$ & $-3.3(<.01)$ & $-6.3(<.001)$ & \\
\hline \multicolumn{7}{|c|}{ Rescue medication at 5 min after blinded esmolol start } \\
\hline Met rescue criterion & & $22(61 \%)$ & $21(49 \%)$ & $20(54 \%)$ & $63(54 \%)$ & $\begin{array}{l}.54 \dagger \\
.56 \dagger\end{array}$ \\
\hline Rescue medication at $5 \mathrm{~min}^{*}$ & & $12(33 \%)$ & $12(28 \%)$ & $15(41 \%)$ & $39(34 \%)$ & $\begin{array}{l}.48 \dagger \\
.54 \dagger\end{array}$ \\
\hline \multicolumn{7}{|l|}{ Type of rescue medication; } \\
\hline Increased isoflurane & & $9(75 \%)$ & $12(100 \%)$ & $13(87 \%)$ & $34(87 \%)$ & \\
\hline Nitroprusside & & $3(25 \%)$ & $1(8 \%)$ & $1(7 \%)$ & $5(13 \%)$ & \\
\hline Fentanyl & & $0(0 \%)$ & $1(8 \%)$ & $1(7 \%)$ & $2(5 \%)$ & \\
\hline Propofol & & $1(8 \%)$ & $0(0 \%)$ & $0(0 \%)$ & $1(3 \%)$ & \\
\hline
\end{tabular}

$S D$, Standard deviation. ${ }^{*}$ Subjects might have received more than 1 rescue medication. $\dagger$ Exact $\chi^{2}$ test. $\dagger$ Cochran-Armitage linear trend test.

\section{Pharmacokinetics}

A total of 107 patients had pharmacokinetic data further analyzed: 22 newborns, 42 infants, and 43 children. The 15-minute arterial plasma esmolol concentration was significantly higher in children compared with that seen in newborns $(P<.0001)$ and infants $(P=.001)$; no difference was detected between newborns and infants. Plasma esmolol concentrations appeared to increase in proportion to dose (Figure 2). The time to steady state did not differ significantly as a function of age, and patients were approaching steady state by 15 minutes.

The elimination rate constant ( \pm standard error) determined across dose levels was $0.146 \pm 0.04\left(r^{2}=0.67\right)$. The time to steady state for the population was 21 minutes, with a half-life of accumulation (equivalent to the half-life of elimination for a drug administered by means of continuous intravenous infusion) of 4.8 minutes. For children, the $\mathrm{C}_{\mathrm{SS}}$ values ( \pm standard error) for the 125,250 , and 500 $\mu \mathrm{g} / \mathrm{kg}$ dose levels were $992 \pm 143,1983 \pm 287$, and 3967 $\pm 573 \mathrm{ng} / \mathrm{mL}$, and for the newborn/infants, the $\mathrm{C}_{\mathrm{SS}}$ values ( \pm standard error) for the 125,250 and $500 \mu \mathrm{g} \cdot \mathrm{kg}^{-1}$. $\min ^{-1}$ dose levels were $444 \pm 60,888 \pm 120$, and $1777 \pm$ $240 \mathrm{ng} / \mathrm{mL}$, respectively.
Esmolol clearance was greater in the newborn/infant group $\left(281 \mathrm{~mL} \cdot \mathrm{kg}^{-1} \cdot \min ^{-1} ; 95 \%\right.$ confidence limit [CL], 267-296 $\mathrm{mL} \cdot \mathrm{kg}^{-1} \cdot \mathrm{min}^{-1}$ ) compared with that for children $\left(126 \mathrm{~mL} \cdot \mathrm{kg}^{-1} \cdot \min ^{-1} ; 95 \% \mathrm{CL}, 83-169 \mathrm{~mL}\right.$. $\left.\mathrm{kg}^{-1} \cdot \min ^{-1}\right)$. Because of the high degree of interpatient variability, individual drug clearances could not be calculated, and thus correlation between age and clearance could not be performed.

\section{Adverse Events}

There were no deaths and no serious AEs. Most of the complications were typical of patients recovering from surgical CoA repair. All 3 dose groups showed similar incidences of AEs. The most frequent AEs were postoperative pain $(n=90[76 \%])$, postoperative agitation ( $n=24$ [20\%]), postoperative anemia $(\mathrm{n}=22[19 \%])$, hypokalemia $(\mathrm{n}=$ 32 [27\%]), and metabolic acidosis ( $n=33$ [28\%]; Table 4). Specific to this patient population were bleeding that required transfusion $(n=35[30 \%])$, pneumothorax $(n=17$ [14\%]), and sequelae associated with the esmolol intravenous infusion site $(\mathrm{n}=1[1 \%])$.

There were 17 AEs that are known therapeutic end points or previously described side effects of esmolol: hypotension

TABLE 3. Change from baseline in heart rate at 5 minutes after the start of esmolol

\begin{tabular}{|c|c|c|c|c|c|}
\hline Parameter & Statistic/category & Low dose & Medium dose & High dose & $P$ value \\
\hline Per-protocol & $\mathrm{N}$ & 33 & 41 & 35 & \\
\hline Change from baseline (beats/min) & $\begin{array}{l}\text { Mean (SD) } \\
95 \% \mathrm{CL}\end{array}$ & $\begin{array}{l}-6.8 \pm 9.9 \\
-10.4 \text { to }-3.3\end{array}$ & $\begin{array}{l}-14.2 \pm 8.5 \\
-17.4 \text { to }-11.0\end{array}$ & $\begin{array}{l}-13.2 \pm 12.7 \\
-16.7 \text { to }-9.7\end{array}$ & .007 \\
\hline Intent to treat & $\mathrm{N}$ & 36 & 31 & 75 & \\
\hline Change from baseline (beats/min) & $\begin{array}{l}\text { Mean (SD) } \\
95 \% \text { CL }\end{array}$ & $\begin{array}{l}-7.4 \pm 9.7 \\
-11.1 \text { to }-3.7\end{array}$ & $\begin{array}{l}-13.2 \pm 10.7 \\
-16.6 \text { to }-9.8\end{array}$ & $\begin{array}{l}-12.3 \pm 12.9 \\
-15.3 \text { to }-8.6\end{array}$ & .06 \\
\hline
\end{tabular}

$S D$, Standard deviation; $C L$, confidence limit. 


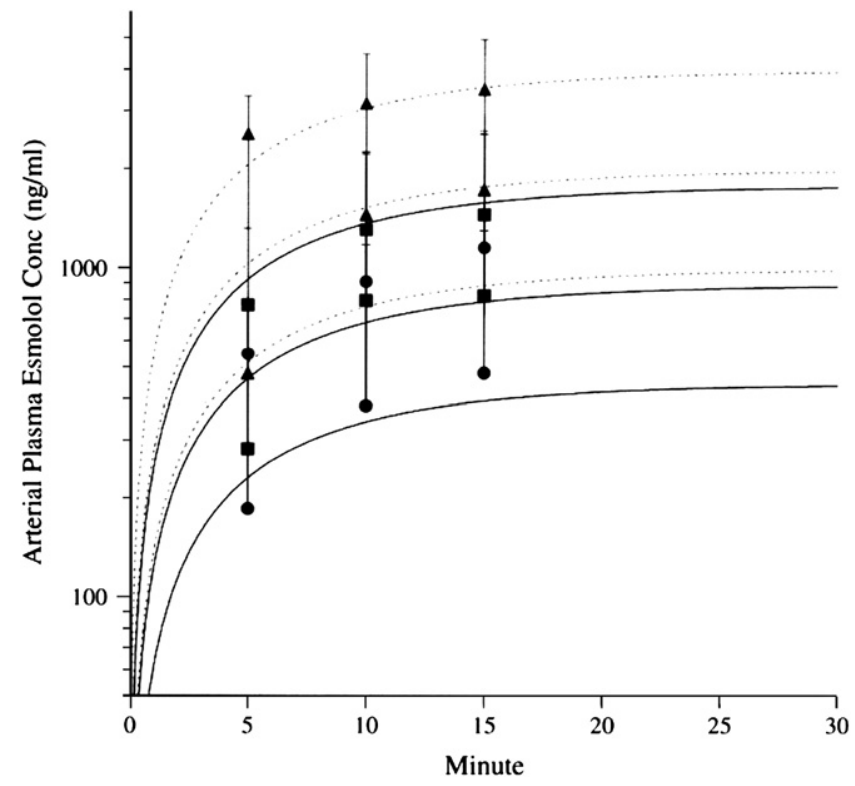

Figure 2. Concentration time curves for the 125 (circles), 250 (squares), and 500 (triangles) $\mu \mathrm{g} \cdot \mathrm{kg}^{-1} \cdot \mathrm{min}^{-1}$ dose levels. The child age group is shown with dotted lines and gray symbols, and the newborn/infant groups are shown with solid lines and black symbols. Mean \pm standard deviation arterial plasma concentrations are shown. Curve fits were derived by using all concentration time point data and not the mean values.

$(\mathrm{n}=10[8 \%])$, bradycardia $(\mathrm{n}=1[1 \%])$, wheezing $(\mathrm{n}=3$ [3\%]), and reaction at the injection site $(n=1[1 \%])$. Seven subjects discontinued the study because of AEs: hypotension $(\mathrm{n}=5)$, bilateral wheezing $(\mathrm{n}=1)$, and reaction at the injection site $(\mathrm{n}=1)$.

\section{Discussion}

CoA can present critically in infancy when the ductus arteriosus closes or later during childhood. The current approach to isolated CoA is either surgical, usually through a thoracotomy, ${ }^{2,3}$ or through interventional catheterization with a balloon angioplasty or intravascular stent. ${ }^{13,14}$

Hypertension occurs in more than $65 \%$ of subjects during the postoperative period after surgical coarctation repair. ${ }^{4-6}$ Theories about the cause of postoperative hypertension include activation of the sympathetic nervous system, the renin-angiotension system, or both. More recent studies implicate abnormalities in the elastic properties of the aorta ${ }^{15}$ and autonomic dysfunction ${ }^{16}$ as causes for postoperative hypertension, particularly in newborns.

The use of $\beta$-blockade (propranolol) in children during the perioperative period for coarctation repair has been shown to result in a significant decrease in SBP and diastolic blood pressure and in plasma renin activity. ${ }^{6}$ Esmolol has several advantages over propranolol: rapid hydrolysis by red blood cell esterases ${ }^{17}$ and selective, competitive $\beta_{1}$-blockade. ${ }^{7}$ Initial pharmacokinetic data examining the use of esmolol in children indicate a faster elimination than reported in adults. ${ }^{8,9,18}$ Esmolol has been shown to control hypertension in children after cardiac surgery ${ }^{9}$ and specifically after CoA repair (in both case reports ${ }^{10}$ and small series ${ }^{9,11}$ ). When used as a single antihypertensive agent, reports indicate that higher doses might be required after CoA repair (830 $\left.\pm 153 \mu \mathrm{g} \cdot \mathrm{kg}^{-1} \cdot \min ^{-1}\right)$ compared with other lesions ${ }^{10}$ and higher doses than suggested in the product label (300 $\left.\mu \mathrm{g} \cdot \mathrm{kg}^{-1} \cdot \min ^{-1}\right)$.

This article reports the first dose-response and safety study of esmolol for the treatment of hypertension in infants and children after CoA repair. The primary aim was to determine the efficacy $(n=116)$ at 3 dose levels of controlling postoperative hypertension within 30 minutes of aortic crossclamp release and at 5 minutes after the initiation of esmolol. The secondary aims were $(1)$ the pharmacokinetics $(n=107)$ of esmolol when administered as a bolus followed by an immediate infusion and $(2)$ the safety $(n=118)$ of esmolol use in this population.

We found a significant decrease in SBP at 5 minutes after initiation of esmolol compared with baseline values in all dose groups, with no significant difference between dose groups. When patients were divided into age groups, there was still no significant difference in the effect on SBP between dose groups. The chosen dosing range (125-500 $\mu \mathrm{g}$ $\left.\cdot \mathrm{kg}^{-1} \cdot \min ^{-1}\right)$ was considered by the advisory committee to be within the standard of care. A placebo control was not believed to provide clinical equipoise. Other studies have suggested that higher doses of esmolol might be necessary when used as a single antihypertensive agent. ${ }^{10} \mathrm{Al}-$ though some patients received higher doses of esmolol (either blinded or open label), it occurred outside the close blood pressure monitoring window of the study and in conjunction with other antihypertensive agents. Thus the effect of higher-dose esmolol could not be evaluated in this study population. It might also be that esmolol in this range is most effective if used in combination with an antihypertensive agent with an alternative mode of action, such as nitroprusside. Finally, it is possible that the incremental difference between dose ranges was too small to result in a statistically significant difference in blood pressure. This is supported by the pharmacokinetic finding of significant interpatient variability within each dose group.

Plasma esmolol concentration increased in proportion to dose over the 125 to $500 \mu \mathrm{g} \cdot \mathrm{kg}^{-1} \cdot \mathrm{min}^{-1}$ dose range. In addition, there was a 4-fold increase in 5-, 10-, and 15-minute plasma drug concentrations, respectively. Although there was significant interpatient variability, the esmolol clearance in children of $126 \mathrm{~mL} \cdot \mathrm{kg}^{-1} \cdot \min ^{-1}$ was similar to that reported in other recent pediatric studies, ${ }^{8,9,19}$ whereas clearance in newborns $\left(281 \mathrm{~mL} \cdot \mathrm{kg}^{-1} \cdot \mathrm{min}^{-1}\right)$ was considerably greater. 
TABLE 4. Adverse events (\%) occurring with an incidence of 10 or greater in the safety dataset*

\begin{tabular}{|c|c|c|c|c|}
\hline System organ class/adverse event & Low dose $(n=36)$ & Medium dose $(n=44)$ & High dose $(n=38)$ & Overall $(n=118)$ \\
\hline \multicolumn{5}{|l|}{ Cardiac disorders } \\
\hline Tachycardia NOS & $3(8)$ & $5(11)$ & $3(8)$ & $11(9)$ \\
\hline \multicolumn{5}{|l|}{ Gastrointestinal disorders } \\
\hline Constipation & $3(8)$ & $2(5)$ & $10(26)$ & $15(13)$ \\
\hline \multicolumn{5}{|l|}{ General disorders } \\
\hline Pyrexia & $3(8)$ & $8(18)$ & $4(11)$ & $15(13)$ \\
\hline \multicolumn{5}{|c|}{ Injury, poisoning, and procedural complications } \\
\hline Agitation postoperative & $6(17)$ & $10(23)$ & $8(21)$ & $24(20)$ \\
\hline Anemia postoperative & $6(17)$ & 8 (18) & $8(21)$ & $22(19)$ \\
\hline Pain postoperative & $27(75)$ & $35(80)$ & $28(74)$ & $90(76)$ \\
\hline Vomiting postoperative & $3(8)$ & $4(9)$ & $5(13)$ & $12(10)$ \\
\hline \multicolumn{5}{|l|}{ Metabolism and nutritional disorders } \\
\hline Hypocalcemia & $3(8)$ & $6(14)$ & $3(8)$ & $12(10)$ \\
\hline Fluid overload & $2(6)$ & $2(5)$ & $6(16)$ & $10(8)$ \\
\hline Hypokalemia & $10(28)$ & $10(23)$ & $12(32)$ & $32(27)$ \\
\hline Metabolic acidosis & $9(25)$ & $12(27)$ & $12(32)$ & $33(28)$ \\
\hline \multicolumn{5}{|l|}{ Renal and urinary disorders } \\
\hline Oliguria & $13(36)$ & $13(30)$ & $8(21)$ & $32(27)$ \\
\hline \multicolumn{5}{|l|}{ Skin and subcutaneous tissue disorders } \\
\hline Subcutaneous emphysema & $1(3)$ & $5(11)$ & $6(16)$ & $12(10)$ \\
\hline \multicolumn{5}{|l|}{ Vascular disorders } \\
\hline Hypotension NOS & $3(8)$ & $3(7)$ & $4(11)$ & $10(8)$ \\
\hline \multicolumn{5}{|l|}{$\begin{array}{l}\text { Respiratory, thoracic, and mediastinal } \\
\text { disorders }\end{array}$} \\
\hline Atelectasis & $3(8)$ & $13(30)$ & $11(28)$ & $27(23)$ \\
\hline Pleural effusion & $2(6)$ & $2(5)$ & $6(16)$ & $10(8)$ \\
\hline Pneumothorax & $4(11)$ & $7(16)$ & $6(16)$ & $17(14)$ \\
\hline Respiratory acidosis & $2(6)$ & $5(11)$ & $4(11)$ & $11(9)$ \\
\hline
\end{tabular}

*If a subject had the same adverse event 2 or more times, the adverse event was only counted once.

There were no mortalities and no serious AEs. The most common AEs were expected after surgical coarctation repair (pain, agitation, and anemia). Despite the maximum esmolol concentration of $20 \mathrm{mg} / \mathrm{mL}$, only 1 patient had irritation at the site of intravenous administration. Because metabolic acidosis $(28 \%)$ and hypokalemia $(27 \%)$ are common after cardiac surgery, in the absence of nonsurgical controls, it is beyond the limits of this study to determine whether esmolol played a role. There was no effect of dose range on the incidence of AEs.

The results of this study demonstrate that esmolol decreases SBP immediately after repair of CoA in infants and children, with no significant untoward events. This study is unique in the fact that it is perioperative and multi-institutional (United States and Canada) and crosses age groups (newborn to 6 years) and disciplines (surgeons, anesthesiologists, and intensivists). The collaboration for this study helped to lay the foundation and infrastructure for current, ongoing, multi-institutional pediatric cardiac trials.

\section{Study Limitations}

Because of concern over the risk of early postoperative bleeding from the aortic suture line, the use of a placebo control was believed to be unsafe. The dose range was limited by current practice in this patient population. The wide age range was chosen to enable an adequate study population. The intraoperative time period within 30 minutes of aortic crossclamp release was chosen to achieve the most uniform patient condition. Thus most patients were still receiving inhaled anesthetic, the vasodilating properties of which might diminish the effect of esmolol. The combination of these factors is the most likely explanation for why the decrease in SBP with esmolol was not statistically significant between dose groups.

Furthermore, pharmacokinetic data analyses found that steady-state esmolol blood levels were achieved 20 minutes after the bolus was administered; however, primary efficacy measures were determined only 5 minutes after the esmolol bolus, perhaps underestimating the optimal effect of esmolol on decreasing blood pressure. 


\section{Conclusions}

Esmolol safely and significantly decreases SBP immediately after surgical repair of CoA in infants and children. However, no difference was found between doses $(125,250$, and 500 $\left.\mu \mathrm{g} \cdot \mathrm{kg}^{-1} \cdot \min ^{-1}\right)$. This study lays the foundation for determining the effect of higher doses of esmolol on blood pressure from both an age-dependent and drug-combination (eg, nitroprusside) approach.

We thank Cheng-Tao Chang, PhD, for his help with the statistics.

\section{References}

1. Samanek M, Slavik Z, Zborilova B, Hrobonova V, Voriskova M, Skovranek J. Prevalence, treatment and outcome of heart disease in live-born children: a prospective analysis of 91,823 live-born children. Pediatr Cardiol. 1989;10:205-11.

2. Wood AE, Javadpour H, Duff D, Oslizlok P, Walsh K. Is extended arch aortoplasty the operation of choice for infant coarctation? Results of 15 years' experience in 181 patients. Ann Thorac Surg. 2004;77:1353-8.

3. Wright GE, Nowak CA, Goldberg CS, Ohye RG, Bove EL, Rocchini AP. Extended resection and end-to-end anastomosis for aortic coarctation in infants: results of a tailored surgical approach. Ann Thorac Surg. 2005;80:1453-9.

4. Sealey WC. Paradoxical hypertension after repair of coarctation of the aorta: a review of its causes. Ann Thorac Surg. 1990;50:323-9.

5. Rocchini AP, Rosenthal A, Barger AC, Castaneda AR, Nadas AS. Pathogenesis of paradoxical hypertension after coarctation resection. Circulation. 1976;54:382-7.

6. Gidding SS, Rocchini AP, Beekman R, Szpunar CA, Moorehead C, Behrendt $\mathrm{D}$, et al. Therapeutic effect of propranolol on paradoxical hypertension after repair of coarctation of the aorta. N Engl J Med. 1985; 312:1224-8

7. Angaran DM, Schultz NJ, Tschida VH. Esmolol hydrochloride: an ultrashort-acting, beta-adrenergic blocking agent. Clin Pharm. 1986;5: 288-303.

\section{Appendix}

\section{Participating Centers}

United States: Children's Hospital of Philadelphia, Philadelphia, Pa, Sarah Tabbutt, MD, PhD, Susan Nicolson, MD; Los Angeles Children's Hospital, Los Angeles, Calif, Winfield Wells, MD; Cleveland Clinic Foundation, Cleveland, Ohio, Paula Bokesch, MD; Children's Hospital of Wisconsin, Milwaukee, Wis, James Tweddell, MD; Children's Hospital Boston, Boston, Mass, Francis McGowan, MD; Children's Memorial Hospital, Chicago, Ill, Carl Backer, MD; Texas Children's Hospital, Houston, Tex, Steven Stayer, MD; Columbus Children's Hospital, Columbus, Ohio, Jill Fitch, MD; Children's Hospital of Cincinnati, Cincinnati, Ohio, David Nelson, MD, PhD; University of Michigan, Ann Arbor, Mich, Thomas Kulik; San Diego Children's Hospital, San Diego, Calif, David Bichell, MD; Hope Children's Hospital, Oak Lawn, Ill, Joanne Starr, MD; Duke University, Durham, NC, Scott Schulman, MD; Montefiore Medical Center, Bronx, NY, Gregory Crooke, MD; Denver Children's Hospital, Denver, Colo, Dunbar Ivy, MD; Children's National Med-
8. Cuneo BF, Zales VR, Blahunka PC, Benson DW. Pharmacodynamics and pharmacokinetics of esmolol, a short-acting beta-blocking agent, in children. Pediatr Cardiol. 1994;15:296-301.

9. Wiest DB, Garner SS, Uber WE, Sade RM. Esmolol for the management of pediatric hypertension after cardiac operations. J Thorac Cardiovasc Surg. 1998;115:890-7.

10. Smerling A, Gersony WM. Esmolol for severe hypertension following repair of aortic coarctation. Crit Care Med. 1990;18:1288-90.

11. Vincent RN, Click LA, Willliams HM, Plauth WH, Williams WH. Esmolol as an adjunct in the treatment of systemic hypertension after operative repair of coarctation of the aorta. Am J Cardiol. 1990;65:941-3.

12. Zuppa AF, Shi H, Adamson PC. Liquid chromatography-electrospray mass spectrometry (LC-MS) method for determination of esmolol concentration in human plasma. J Chromatogr B Analyt Technol Biomed Life Sci. 2003;792:203-301.

13. Cowley CG, Orsmond GS, Feola P, McQuillan L, Shaddy RE. Longterm, randomized comparison of balloon angioplasty and surgery for native coarctation of the aorta in childhood. Circulation. 2005;111:3453-6.

14. Walhout RJ, Lekkerkerker JC, Oron GH, Bennink GBWE, Meijboom EJ. Comparison of surgical repair with balloon angioplasty for native coarctation in patients from 3 months to 16 years of age. Eur J Cardiothorac Surg. 2004;25:722-7.

15. Vogt M, Kuhn A, Baumgartner D, Baumgartner C, Busch R, Kostolny M, et al. Impaired elastic properties of the ascending aorta in newborns before and early after successful coarctation repair. Proof of a systemic vascular disease of the prestenotic arteries. Circulation. 2005; 111:3269-73.

16. Polson JW, McCallion N, Waki H, Thorne G, Tooley MA, Paton JFR, et al. Evidence of cardiovascular autonomic dysfunction in neonates with coarctation of the aorta. Circulation. 2006;113:2844-50.

17. Reynolds RD, Gorczynski RJ, Quon CY. Pharmacology and pharmacokinetics of esmolol. J Clin Pharmacol. 1986;26A:A3-14.

18. Wiest DB, Trippel DL, Gillette PC, Garner SS. Pharmacokinetics of esmolol in children. Clin Pharmacol Ther. 1991;49:618-23.

19. Adamson PC, Rhodes LA, Saul JP, Dick M, Epstein MR, Moate P, et al. The pharmacokinetics of esmolol in pediatric subjects with supraventricular arrhythmias. Pediatr Cardiol. 2006;27:420-7.

ical Center, Washington, DC, Eric Quivers, MD; University of Iowa, Iowa City, Iowa, Javier Campos, MD; Children's Hospital of Pittsburg, Pittsburg, Pa, Gerard Boyle, MD.

Canada: British Columbia Children's Hospital, Vancouver, British Columbia, Suvro Sett, MD; Hospital for Sick Children, Toronto, Ontario, Glen Van Arsdell, MD.

\section{Steering Committee}

Children's Hospital of Philadelphia: S. Tabbutt, S. Nicolson, M. Schreiner, P. Adamson, A. Cnaan; Boston Children's Hospital: F. McGowan; Cleveland Clinic: P. Bokesch; Baxter Healthcare Corporation: M. Hoffman, K. Allenby, P. Jambhekar, C. T. Chang.

\section{Data and Safety Monitoring IND Safety}

Reports for the multicenter trial were submitted by Baxter Healthcare Corporation to the FDA and also all institutional review boards. 